\title{
Review Article \\ Genetic Contribution to the Pathogenesis of Primary Biliary Cholangitis
}

\author{
Satoru Joshita, ${ }^{1}$ Takeji Umemura, ${ }^{1}$ Eiji Tanaka, ${ }^{1}$ and Masao Ota ${ }^{2}$ \\ ${ }^{1}$ Department of Medicine, Division of Gastroenterology and Hepatology, Shinshu University School of Medicine, \\ 3-1-1 Asahi, Matsumoto, Nagano 390-8621, Japan \\ ${ }^{2}$ Department of Legal Medicine, Shinshu University School of Medicine, 3-1-1 Asahi, Matsumoto, Nagano 390-8621, Japan
}

Correspondence should be addressed to Satoru Joshita; joshita@shinshu-u.ac.jp and Masao Ota; otamasao@shinshu-u.ac.jp

Received 18 October 2016; Accepted 12 January 2017; Published 1 February 2017

Academic Editor: Nancy Agmon-Levin

Copyright (C) 2017 Satoru Joshita et al. This is an open access article distributed under the Creative Commons Attribution License, which permits unrestricted use, distribution, and reproduction in any medium, provided the original work is properly cited.

\begin{abstract}
Formerly termed primary biliary cirrhosis, primary biliary cholangitis (PBC) is a chronic and progressive cholestatic liver disease characterized by the presence of antimitochondrial antibodies. Ursodeoxycholic acid (UDCA) therapy is the most effective and approved treatment for PBC and leads to a favorable outcome in the vast majority of cases. Although the etiology of PBC has not yet been elucidated, human leukocyte antigen (HLA) class II alleles have been consistently associated with disease onset for decades. Individuals in different geographic regions of the world may have varying susceptibility alleles that reflect indigenous triggering antigens. In this review, we describe the influence of HLA alleles and other gene polymorphisms on PBC along with the results of genome-wide association studies (GWAS) on this disease.
\end{abstract}

\section{Introduction}

Primary biliary cholangitis (PBC), formerly known as primary biliary cirrhosis $[1,2]$, is a liver-specific autoimmune disease characterized by female preponderance and destruction of intrahepatic bile ducts that often results in cirrhosis and hepatic failure [3-5]. The prevalence of $\mathrm{PBC}$ ranges from 20 to 40 cases per 100,000 persons [4-6], although the number of patients with $\mathrm{PBC}$, specifically asymptomatic $\mathrm{PBC}$, is on the rise due mainly to increased awareness and earlier detection by disease-specific antimitochondrial antibodies (AMAs). Ursodeoxycholic acid (UDCA) therapy is the most effective treatment for PBC and is recommended by most guidelines $[7,8]$. The vast majority of patients with $\mathrm{PBC}$ show a favorable response to UDCA treatment despite some cases of disease progression via unknown mechanisms $[9,10]$. Genetic factors are considered to play a prominent role in disease onset as higher concordance rates in monozygotic twins than in dizygotic twins and familial clustering of patients with $\mathrm{PBC}$ has been demonstrated in family and population studies [11-16]. However, the etiology of this disease has yet to be conclusively clarified; $\mathrm{PBC}$ is presumed to be a multifactorial polygenic condition caused by allelic triggers and environmental factors in genetically susceptible individuals, although epigenetic mechanisms, such as instability of X chromosome gene expression, may also participate in the disease's female predominance [17-19].

In the present article, we summarize the literature on human leukocyte antigen (HLA) involvement in PBC onset and GWAS findings from North American, European, and Japanese populations to explore the disease pathways of PBC pathogenesis.

\section{Associations between HLA and PBC Susceptibility}

Many significant susceptibility single nucleotide polymorphisms (SNPs), such as CTLA4, TNF- $a$, STAT4, PTPN22, and $V D R$, have been identified using candidate gene methods [20-24]. Among them, however, only HLA has consistently been associated with $\mathrm{PBC}$ in distinct patient cohorts across ethnicities.

Located on the most gene-dense genomic region on chromosomal position 6p21 [25], HLA genes are extremely polymorphic and play an essential role in numerous biologically and medically relevant processes. The products of the 
TABLE 1: HLA haplotype associations with PBC.

\begin{tabular}{|c|c|c|c|c|}
\hline Study & Population & HLA allele & $p$ value & OR $(95 \% \mathrm{CI})$ \\
\hline \multicolumn{5}{|l|}{ Susceptibility } \\
\hline \multirow{2}{*}{ Umemura et al. [26] } & \multirow{2}{*}{ Japanese } & DRB1* $^{*} 08: 03-D Q B 1{ }^{*}$ 06:01 & 0.000025 & $2.22(1.53-3.20)$ \\
\hline & & $\mathrm{DRB1}^{*}$ 04:05-DQB1 ${ }^{*}$ 04:01 & 0.044 & $1.38(10.2-1.87)$ \\
\hline \multirow{2}{*}{ Zhao et al. [27] } & \multirow{2}{*}{ Chinese } & $\mathrm{DRB1}^{*}$ 08:03-DQB1* 06:01 & $<0.0001$ & $3.17(1.91-5.23)$ \\
\hline & & $\mathrm{DRB1}^{*}$ 07:01-DQB1*02:02 & 0.005 & $1.85(1.20-2.83)$ \\
\hline \multirow{2}{*}{ Donaldson et al. [28] } & UK & $\mathrm{DRB1}^{*}$ 08:01-DQA1* $04^{*}$ 01-DQB1*04:02 & 0.0027 & 2.9 \\
\hline & Italian & $\mathrm{DRB1}^{*}$ 08:01-DQA1* $04^{*}$ 01-DQB1 ${ }^{*}$ 04:02 & 0.0086 & 3.41 \\
\hline \multicolumn{5}{|l|}{ Protective } \\
\hline \multirow{2}{*}{ Umemura et al. [26] } & \multirow{2}{*}{ Japanese } & DRB1 $^{*} 13: 02-\mathrm{DQB1}{ }^{*}$ 06:04 & 0.00093 & $0.27(0.12-0.60)$ \\
\hline & & DRB1 $^{*}$ 11:01-DQB1* 03:01 & 0.03 & $0.37(0.15-0.88)$ \\
\hline Zhao et al. [27] & Chinese & $\mathrm{DRB1}^{*}$ 12:02-DQB1* $03: 01$ & 0.015 & $0.43(0.22-0.86)$ \\
\hline \multirow{2}{*}{ Donaldson et al. [28] } & UK & DRB1* $^{*}$ 11:01-DQA1* 05:01-DQB1* 03:01 & 0.086 & 0.47 \\
\hline & Italian & DRB1*13:01-DQA1*01:03-DQB1*06:03 & 0.0041 & 0.28 \\
\hline
\end{tabular}

classical HLA class I $(A, B$, and $C)$ and class II $(D R, D Q$, and $D P$ ) genes include cell-surface glycoproteins involved in the binding and presentation of self- or non-self-peptides to Tcell receptors (TCRs). Class I molecules present endogenous peptides derived from viruses to $\mathrm{CD}^{+}$cytotoxic $\mathrm{T}$ cells, while class II molecules present processed peptides from exogenous pathogens to $\mathrm{CD}^{+}$helper $\mathrm{T}$ cells. The extent of endogenous and exogenous peptide binding to HLA molecules depends on allelic polymorphisms. Additionally, both HLA class I and II molecules have functional roles in protein interactions, transcription regulation involved in the inflammatory response, and natural killer cell-cytokine interactions as part of innate immunity.

HLA polymorphisms have been extensively studied in immune-mediated diseases, revealing associations of particular alleles with ankylosing spondylitis (AS), Behçet's disease (BD), psoriasis, multiple sclerosis (MS), insulindependent diabetes mellitus (IDDM), systemic lupus erythematosus (SLE), inflammatory bowel disease (IBD), rheumatoid arthritis (RA), narcolepsy, autoimmune hepatitis (AIH), and autoimmune pancreatitis (AIP) among others. Early investigations on associations between HLA polymorphisms and $\mathrm{PBC}$ were carried out more than a quarter-century ago [37]. Based on these findings, subsequent cumulative studies have provided evidence that $\mathrm{PBC}$ is associated with $D R B 1^{*} 08$ as predisposing and $D R B 1^{*} 11$ and $D R B 1^{*} 13$ as protective alleles $[28,38]$. Li et al. conducted a meta-analysis to assess for relationships between HLA class II and disease susceptibility to $\mathrm{PBC}$ and demonstrated that HLA DR ${ }^{*} 07$ and *08 alleles were risk factors for $\mathrm{PBC}$ in certain populations, whereas $D R^{*} 11,{ }^{*} 12,{ }^{*} 13$, and ${ }^{*} 15$ alleles were protective factors [39].

Several key reports [26-28] on the association between HLA haplotype and PBC susceptibility or resistance are summarized in Table 1. HLA $D R^{*} 08$ alleles caused disease susceptibility, while HLA DRB1 ${ }^{*} 13$ and ${ }^{*} 11$ alleles conferred disease protection in haplotype analyses across ethnicities. Both protective $D R B 1^{*} 11$ and $D R B 1^{*} 13$ alleles have also been implicated $D R B 1^{*} 11$ against hepatitis C [40], human papilloma [41], and human immunodeficiency [42] and $D R B 1^{*} 13$ against hepatitis C [43], human papilloma [44], and human immunodeficiency [45] viruses along with malaria [44]. Thus, one of the pathogenic mechanisms in PBC may be bacterial infection as these protective HLA class II alleles play a functional role in blocking the invasion of infectious agents.

However, individuals harboring the above haplotypes constitute only a minority of patients with $\mathrm{PBC}$, suggesting that other candidate genes and environmental cues evoke PBC pathogenesis. Umemura et al. [26] reported the possibility that the distribution of DRB1 amino acid residues encoded by different HLA DRB1 alleles influenced the binding affinity to antigens, which might also be a predominant factor in $\mathrm{PBC}$ susceptibility.

\section{GWAS on PBC}

There have been extensive GWAS in patients with PBC, a number of which documenting significant associations with disease risk. To date, five GWAS [29-33], two Illumina immunochip studies [34, 35], and one genome-wide metaanalysis (GWMA) [36] on PBC have been performed on well-characterized cohorts in North American, European, and Japanese populations (Table 2). These investigations clarified that the HLA class II domain possessed the strongest association with disease susceptibility, particularly at the HLA-DRB1, HLA-DQA1, and HLA-DQB1 loci. However, HLA alone does not explain the entire genetic predisposition to PBC, mainly since $80-90 \%$ of patients with the disease do not carry the most common HLA susceptibility alleles. In this regard, other genes apart from HLA loci are suggested to contribute to disease development. At present, GWAS have identified 39 non-HLA loci predisposing to PBC at a genomewide level of significance (Table 3 ).

The first GWAS [29] in a North American cohort identified a significant association of $\mathrm{PBC}$ with genetic variants at IL12A, encoding IL-12 p35, and IL12RB2, encoding IL-12 receptor $\beta 2$. Modest $\left(p<5.0 \times 10^{-5}\right)$ genome-wide associations with disease risk for SNPs at the signal transducer 
TABLE 2: GWAS on PBC.

\begin{tabular}{lcccc}
\hline Study & Year & Platform & Patients & Controls \\
\hline Hirschfield et al. [29] & 2009 & Illumina HumanHap 370 & 1,031 & 1,351 \\
Hirschfield et al. [30] & 2010 & Illumina HumanHap 370 & 945 & 4,700 \\
Liu et al. [31] & 2010 & Illumina 610K & 1,840 & 4,651 \\
Mells et al. [32] & 2011 & Illumina 660W-Quad & 1,274 & 5,163 \\
Nakamura et al. [33] & 2012 & Affymetrix Axiom & 2,426 & 1,091 \\
Juran et al. [34] & 2012 & Immunochip & 2,861 & 5,731 \\
Liu et al. [35] & 2012 & Immunochip & 2,764 & 8,514 \\
Cordell et al. [36] & 2015 & GWMA & 10,475 \\
\hline
\end{tabular}

TABLE 3: Non-HLA risk loci identified through GWAS as associated with PBC at the genome-wide level of significance.

\begin{tabular}{|c|c|c|c|c|c|c|c|}
\hline Chromosome & Locus & Study [reference \#] & SNP & OR & $p$ value & Candidate gene(s) & Disease(s) with shared risk loci \\
\hline \multirow{3}{*}{1} & $1 \mathrm{p} 31$ & [35] & rs72678531 & 1.61 & $2.47 E-38$ & IL12RB2 & $\mathrm{BD}$ \\
\hline & $1 \mathrm{p} 36$ & [30] & rs3748816 & 1.33 & $3.15 E-08$ & MMEL1 & MS \\
\hline & $1 \mathrm{q} 31$ & [32] & rs12134279 & 1.34 & $2.06 E-14$ & DENND1B & $\mathrm{CD}$ \\
\hline \multirow{4}{*}{2} & $2 q 12$ & [36] & rs12712133 & 1.14 & $5.19 E-09$ & IL1RL1, IL1RL2, & \multirow{4}{*}{ RA, SLE, Sjögren's, IBD, SSc, BD } \\
\hline & 2q12 & {$[34]$} & rs10186746 & 1.21 & $2.40 E-05$ & IL1RL1, IL1RL2, & \\
\hline & $2 \mathrm{q} 32$ & {$[32]$} & rs10931468 & 1.50 & $2.35 E-19$ & STAT4, STAT1 & \\
\hline & $2 q 36$ & {$[36]$} & rs4973341 & 1.22 & $2.34 E-10$ & CCL20 & \\
\hline \multirow{3}{*}{3} & $3 \mathrm{p} 24$ & {$[32]$} & rs1372072 & 1.20 & $2.28 E-08$ & PLCL2 & RA \\
\hline & $3 q 13$ & [35] & rs2293370 & 1.39 & $6.84 E-16$ & CD80 & MS, SLE, Celiac \\
\hline & $3 q 25$ & [35] & rs2366643 & 1.35 & $3.92 E-22$ & IL12A & Celiac \\
\hline \multirow{2}{*}{4} & $4 \mathrm{p} 16$ & {$[36]$} & rs11724804 & 1.22 & $9.01 E-12$ & DGKQ & \multirow[b]{2}{*}{ UC } \\
\hline & $4 \mathrm{q} 24$ & [32] & rs7665090 & 1.26 & $8.48 E-14$ & NFKB1 & \\
\hline \multirow{3}{*}{5} & $5 \mathrm{p} 13$ & {$[35]$} & rs6871748 & 1.30 & $2.26 E-13$ & IL7R & \multirow[t]{3}{*}{ MS, UC } \\
\hline & $5 q 21$ & [36] & rs526231 & 1.15 & $1.14 E-08$ & C5orf30 & \\
\hline & $5 q 33$ & [36] & rs2546890 & 1.15 & $1.06 E-10$ & IL12B, LOC285626 & \\
\hline \multirow{2}{*}{6} & $6 q 23$ & {$[36]$} & rs6933404 & 1.18 & $1.27 E-10$ & OLIG3, TNFAIP3 & \\
\hline & $6 \mathrm{q} 23$ & {$[34]$} & rs6920220 & 1.29 & $1.17 E-06$ & OLIG3, TNFAIP3 & \\
\hline \multirow{2}{*}{7} & 7 p14 & [32] & rs6974491 & 1.25 & $4.44 E-08$ & ELMO1 & RA, Celiac \\
\hline & $7 \mathrm{q} 32$ & [35] & rs35188261 & 1.52 & $6.52 E-22$ & IRF5 & RA, SLE, SSc, UC \\
\hline 8 & $8 q 24$ & [34] & rs2608029 & 1.23 & $3.14 E-06$ & PVT1, GSDMC & \\
\hline 9 & $9 \mathrm{p} 32$ & {$[33]$} & rs4979462 & 1.57 & $1.85 E-14$ & TNFSF15 & $\mathrm{UC}, \mathrm{CD}$ \\
\hline \multirow{4}{*}{11} & $11 q 13$ & {$[32]$} & rs538147 & 1.23 & $2.06 E-10$ & RPS6KA4 & IBD \\
\hline & $11 q 13$ & [34] & rs10898201 & 1.31 & $4.91 E-06$ & NADSYN1 & \\
\hline & $11 \mathrm{q} 23$ & [33] & rs4938534 & 1.38 & $3.27 E-08$ & POU2AF1 & \\
\hline & $11 \mathrm{q} 23$ & [35] & rs80065107 & 1.39 & $7.20 E-16$ & CXCR5, DDX6 & RA, IBD, Celiac \\
\hline \multirow{3}{*}{12} & $12 \mathrm{p} 13$ & {$[35]$} & rs1800693 & 1.27 & $1.18 E-14$ & TNFRSF1A, LTBR & MS \\
\hline & $12 \mathrm{q} 24$ & [35] & rs11065979 & 1.20 & $2.87 E-09$ & SH2B3 & RA, T1DM, Hyperthyroidism, Celiac \\
\hline & $12 \mathrm{q} 24$ & {$[34]$} & rs7309325 & 1.26 & $2.54 E-05$ & SH2B3 & RA, T1DM, Hyperthyroidism, Celiac \\
\hline 13 & $13 \mathrm{q} 14$ & {$[34,35]$} & rs3862738 & 1.33 & $2.18 E-08$ & TNFSF11 & $\mathrm{CD}$ \\
\hline \multirow{2}{*}{14} & $14 \mathrm{q} 24$ & {$[35]$} & rs911263 & 1.26 & $9.95 E-11$ & RAD51B & RA \\
\hline & $14 \mathrm{q} 32$ & {$[32]$} & rs8017161 & 1.22 & $2.61 E-13$ & TNFAIP2 & \\
\hline \multirow{2}{*}{16} & $16 \mathrm{p} 13$ & {$[35]$} & rs12708715 & 1.29 & $2.19 E-13$ & CLEC16A, SOCS1 & MS, UC, T1DM \\
\hline & $16 \mathrm{q} 24$ & [32] & rs11117432 & 1.31 & $4.66 E-11$ & IRF8 & MS, IBD, RA, SSc \\
\hline \multirow{2}{*}{17} & $17 q 12$ & [35] & rs17564829 & 1.26 & $6.05 E-14$ & IKZF3 & UC, CD, RA, T1DM \\
\hline & $17 q 21$ & [35] & rs17564829 & 1.25 & $2.15 E-09$ & MAPT & \\
\hline \multirow{3}{*}{19} & $19 \mathrm{p} 12$ & {$[35]$} & rs34536443 & 1.91 & $1.23 E-12$ & TYK2 & IBD, RA, SLE, psoriasis, T1DM \\
\hline & $19 \mathrm{p} 13$ & {$[34]$} & rs73003205 & 1.35 & $1.43 E-05$ & KIAA1683 & \\
\hline & $19 \mathrm{q} 13$ & {$[31]$} & rs3745516 & 1.46 & $7.97 E-11$ & SPIB & \\
\hline 22 & $22 \mathrm{q} 13$ & [35] & rs2267407 & 1.29 & $1.29 E-13$ & SYNGR1 & \\
\hline
\end{tabular}

CD, Crohn's disease; UC, ulcerative colitis; T1DM, type 1 diabetes mellitus. 
and activator of transcription 4 (STAT4) and cytotoxic Tlymphocyte-associated protein 4 (CTLA4) loci were found as well. The second GWAS [31] confirmed the existence of additional risk loci, including interferon regulatory factor 5 (IRF5), transportin 3 (TNPO3), and SPIB encoding a transcription factor involved in B-cell receptor signaling and T-cell lineage decisions. A subsequent noteworthy GWAS from Japan showed that the IL12A and IL12RB2 loci were not significantly associated with $\mathrm{PBC}$, but rather that the TNFSF15 and POU2AF1 genes constituted novel risk loci in Japanese patients with $\mathrm{PBC}$ along with other non-HLA loci, including IL7R, IKZF3, CD80, STAT4, and NFKB1. This discrepancy among ethnicities indicated important differences in the pathogenesis of PBC despite several common key molecules and pathways, such as the IL-12 pathway to induce Th1 polarization of $\mathrm{CD}^{+}{ }^{+} \mathrm{T}$ cells. Our body of evidence suggests that there may be an inherited abnormality in immune regulation during $\mathrm{PBC}$ onset and perhaps an inability to suppress inflammatory attacks on small bile ducts once initiated.

It should be noted that Juran et al. identified riskconferring epistatic interactions between IL12RB2 and IRF5 loci [34] as well as between CTLA4 and TNF $\alpha$ loci in the preGWAS era [46]. Epistatic interactions between genes revealed by GWAS in the pathogenesis of PBC should be explored in future studies.

While gene associations are of considerable interest in the pathogenesis of $\mathrm{PBC}$, virtually none have been translated into useful clinical testing. For instance, the importance of the IL-12 pathway in PBC onset has been highlighted in animal models and in the case of a child with a congenital IL-12 deficiency who developed PBC [47]. Although antibodies or drugs targeting the IL-12 pathway would seem to be effective, clinical trials using ustekinumab, a human monoclonal antibody directed against IL-12 and IL-23, have failed to produce effects in phase II trials [48]. One reason explaining the discrepancy between GWAS results and clinical testing may be that clinicians typically encounter patients who have already become complicated with cholestasis; in fact, the immunological destruction of cholangiocytes occurs in the very early stages of $\mathrm{PBC}$. Thus, the mechanisms of disease progression should also be addressed to halt the deterioration of disease status and afford PBC patients an improved prognosis.

Lastly, it is particularly interesting that many genes implicated in PBC pathogenesis by GWAS have also been reported in other autoimmune diseases, such as SLE, systemic sclerosis (SSc), and Sjögren's syndrome (Table 3), suggesting a genetic overlap. Understanding the mechanisms involved in the onset and progression of certain autoimmune diseases may accordingly shed light on those in PBC.

\section{Conclusions and Future Directions}

The pathogenesis of PBC is incompletely understood but appears to involve genetic susceptibility and resistance alleles in HLA and other gene loci, with a possible overlap with several autoimmune diseases. It is also probable that genetically susceptible individuals develop PBC following environmental cues, leading to both adaptive and innate immune responses that result in portal inflammation and bile duct epithelial damage. In addition to susceptibility, the precise mechanisms of $\mathrm{PBC}$ progression should be addressed to improve patient prognosis and quality of life.

\section{Competing Interests}

The authors declare that they have nothing to disclose regarding funding from industries or conflict of interests with respect to this manuscript.

\section{Acknowledgments}

The authors sincerely appreciate the financial support provided by Grants-in-Aid for Young Scientists (Kakenhi 16K21069) and the Promotion Project of Education, Research, and Medical Care from Shinshu University Hospital. They also thank Mr. Trevor Ralph for his English editorial assistance.

\section{References}

[1] U. Beuers, M. E. Gershwin, R. G. Gish et al., "Changing nomenclature for PBC: from 'cirrhosis' to 'cholangitis,' Gastroenterology, vol. 149, no. 6, pp. 1627-1629, 2015.

[2] A. Tanaka, H. Takikawa, H. Miwa, T. Shimosegawa, S. Mochida, and K. Koike, "Changing nomenclature for $\mathrm{PBC}$ from 'primary biliary cirrhosis' to'primary biliary cholangitis,' Hepatology Research, vol. 46, no. 8, pp. 725-726, 2016.

[3] J. A. Talwalkar and K. D. Lindor, "Primary biliary cirrhosis," Lancet, vol. 362, no. 9377, pp. 53-61, 2003.

[4] W. R. Kim, K. D. Lindor, G. R. Locke et al., "Epidemiology and natural history of primary biliary cirrhosis in a U.S. community," Gastroenterology, vol. 119, no. 6, pp. 1631-1636, 2000.

[5] K. D. Lindor, M. E. Gershwin, R. Poupon, M. Kaplan, N. V. Bergasa, and E. J. Heathcote, "Primary biliary cirrhosis," Hepatology, vol. 50, no. 1, pp. 291-308, 2009.

[6] S. Toshihito, O. Kazuichi, H. Kenichi, I. Hiromi, N. Yasuni, and T. Hirohito, Epidemiology and Natural History in Japan, Springer, Tokyo, Japan, chaper 15 edition, 2014.

[7] European Association for the Study of the Liver, "EASL clinical practice guidelines: management of cholestatic liver diseases," Journal of Hepatology, vol. 51, pp. 237-267, 2009.

[8] A. Komori, A. Tanaka, H. Takikawa et al., "Guidelines for the management of primary biliary cirrhosis: The Intractable Hepatobiliary Disease Study Group supported by the Ministry of Health, Labour and Welfare of Japan," Hepatology Research, vol. 44, Supplement 1, pp. 71-90, 2014.

[9] S. Joshita, T. Umemura, M. Ota, and E. Tanaka, "AST/platelet ratio index associates with progression to hepatic failure and correlates with histological fibrosis stage in Japanese patients with primary biliary cirrhosis," Journal of Hepatology, vol. 61, no. 6, pp. 1443-1445, 2014.

[10] T. Umemura, S. Joshita, T. Sekiguchi et al., "Serum Wisteria floribunda agglutinin-positive Mac-2-binding protein level predicts liver fibrosis and prognosis in primary biliary cirrhosis," American Journal of Gastroenterology, vol. 110, no. 6, pp. 857864, 2015. 
[11] D. E. J. Jones, "Pathogenesis of primary biliary cirrhosis," Journal of Hepatology, vol. 39, no. 4, pp. 639-648, 2003.

[12] M. E. Gershwin, C. Selmi, H. J. Worman et al., "Risk factors and comorbidities in primary biliary cirrhosis: a controlled interview-based study of 1032 patients," Hepatology, vol. 42, no. 5, pp. 1194-1202, 2005.

[13] M. M. Kaplan and M. E. Gershwin, "Primary biliary cirrhosis," New England Journal of Medicine, vol. 353, no. 12, pp. 1261-1273, 2005.

[14] M. E. Gershwin and I. R. Mackay, "The causes of primary biliary cirrhosis: convenient and inconvenient truths," Hepatology, vol. 47, no. 2, pp. 737-745, 2008.

[15] P. Invernizzi, C. Selmi, I. R. Mackay, M. Podda, and M. E. Gershwin, "From bases to basis: linking genetics to causation in primary biliary cirrhosis," Clinical Gastroenterology and Hepatology, vol. 3, no. 5, pp. 401-410, 2005.

[16] C. Selmi, M. J. Mayo, N. Bach et al., "Primary biliary cirrhosis in monozygotic and dizygotic twins: genetics, epigenetics, and environment," Gastroenterology, vol. 127, no. 2, pp. 485-492, 2004.

[17] P. Invernizzi, M. Miozzo, P. M. Battezzati et al., "Frequency of monosomy $\mathrm{X}$ in women with primary biliary cirrhosis," Lancet, vol. 363, no. 9408, pp. 533-535, 2004.

[18] M. Miozzo, C. Selmi, B. Gentilin et al., "Preferential X chromosome loss but random inactivation characterize primary biliary cirrhosis," Hepatology, vol. 46, no. 2, pp. 456-462, 2007.

[19] Y.-Q. Xie, H.-D. Ma, and Z.-X. Lian, "Epigenetics and primary biliary cirrhosis: a comprehensive review and implications for autoimmunity," Clinical Reviews in Allergy and Immunology, vol. 50, no. 3, pp. 390-403, 2016.

[20] B. D. Juran, E. J. Atkinson, E. M. Schlicht, B. L. Fridley, and K. N. Lazaridis, "Primary biliary cirrhosis is associated with a genetic variant in the $3^{\prime}$ flanking region of the CTLA4 gene," Gastroenterology, vol. 135, no. 4, pp. 1200-1206, 2008.

[21] A. Tanaka, S. Nezu, S. Uegaki et al., "Vitamin D receptor polymorphisms are associated with increased susceptibility to primary biliary cirrhosis in Japanese and Italian populations," Journal of Hepatology, vol. 50, no. 6, pp. 1202-1209, 2009.

[22] S. Joshita, T. Umemura, K. Yoshizawa et al., "Association analysis of cytotoxic T-lymphocyte antigen 4 gene polymorphisms with primary biliary cirrhosis in Japanese patients," Journal of Hepatology, vol. 53, no. 3, pp. 537-541, 2010.

[23] S. Joshita, T. Umemura, M. Nakamura et al., "STAT4 gene polymorphisms are associated with susceptibility and ANA status in primary biliary cirrhosis," Disease Markers, vol. 2014, Article ID 727393, 8 pages, 2014.

[24] T. Umemura, S. Joshita, T. Yamazaki et al., "Genetic Association of PTPN22 Polymorphisms with Autoimmune Hepatitis and Primary Biliary Cholangitis in Japan," Scientific Reports, vol. 6, Article ID 29770, 2016.

[25] T. Umemura and M. Ota, "Genetic factors affect the etiology, clinical characteristics and outcome of autoimmune hepatitis," Clinical Journal of Gastroenterology, vol. 8, no. 6, pp. 360-366, 2015.

[26] T. Umemura, S. Joshita, T. Ichijo et al., "Human leukocyte antigen class II molecules confer both susceptibility and progression in Japanese patients with primary biliary cirrhosis," Hepatology, vol. 55, no. 2, pp. 506-511, 2012.

[27] D.-T. Zhao, H.-Y. Liao, X. Zhang et al., "Human leucocyte antigen alleles and haplotypes and their associations with antinuclear antibodies features in Chinese patients with primary biliary cirrhosis," Liver International, vol. 34, no. 2, pp. 220-226, 2014.

[28] P. T. Donaldson, A. Baragiotta, M. A. Heneghan et al., "HLA class II alleles, genotypes, haplotypes, and amino acids in primary biliary cirrhosis: a large-scale study," Hepatology, vol. 44, no. 3, pp. 667-674, 2006.

[29] G. M. Hirschfield, X. Liu, C. Xu et al., "Primary biliary cirrhosis associated with HLA, IL12A, and IL12RB2 variants," New England Journal of Medicine, vol. 360, no. 24, pp. 2544$2555,2009$.

[30] G. M. Hirschfield, X. Liu, Y. Han et al., "Variants at IRF5TNPO3, 17q12-21 and MMEL1 are associated with primary biliary cirrhosis," Nature Genetics, vol. 42, no. 8, pp. 655-657, 2010.

[31] X. Liu, P. Invernizzi, Y. Lu et al., "Genome-wide meta-analyses identify three loci associated with primary biliary cirrhosis," Nature Genetics, vol. 42, no. 8, pp. 658-660, 2010.

[32] G. F. Mells, J. A. B. Floyd, K. I. Morley et al., "Genomewide association study identifies 12 new susceptibility loci for primary biliary cirrhosis," Nature Genetics, vol. 43, no. 4, pp. 329-332, 2011.

[33] M. Nakamura, N. Nishida, M. Kawashima et al., "Genomewide association study identifies TNFSF15 and POU2AF1 as susceptibility loci for primary biliary cirrhosis in the Japanese population," American Journal of Human Genetics, vol. 91, no. 4, pp. 721-728, 2012.

[34] B. D. Juran, G. M. Hirschfield, P. Invernizzi et al., "Immunochip analyses identify a novel risk locus for primary biliary cirrhosis at 13q14, multiple independent associations at four established risk loci and epistasis between 1p31 and 7q32 risk Variants," Human Molecular Genetics, vol. 21, no. 23, pp. 5209-5221, 2012.

[35] J. Z. Liu, M. A. Almarri, D. J. Gaffney et al., "Dense finemapping study identifies new susceptibility loci for primary biliary cirrhosis," Nature Genetics, vol. 44, no. 10, pp. 1137-1141, 2012.

[36] H. J. Cordell, Y. Han, G. F. Mells et al., "International genomewide meta-analysis identifies new primary biliary cirrhosis risk loci and targetable pathogenic pathways," Nature Communications, vol. 6, article 8019, 2015.

[37] G. Ercilla, A. Pares, F. Arriaga et al., "Primary biliary cirrhosis associated with HLA-DRw3," Tissue Antigens, vol. 14, no. 5, pp. 449-452, 1979.

[38] P. Invernizzi, C. Selmi, F. Poli et al., "Human leukocyte antigen polymorphisms in Italian primary biliary cirrhosis: a multicenter study of 664 patients and 1992 healthy controls," Hepatology, vol. 48, no. 6, pp. 1906-1912, 2008.

[39] M. Li, H. Zheng, Q.-B. Tian, M.-N. Rui, and D.-W. Liu, "HLADR polymorphism and primary biliary cirrhosis: evidence from a meta-analysis," Archives of Medical Research, vol. 45, no. 3, pp. 270-279, 2014.

[40] L. Alric, M. Fort, J. Izopet et al., "Genes of the major histocompatibility complex class II influence the outcome of hepatitis C virus infection," Gastroenterology, vol. 113, no. 5, pp. 1675-1681, 1997.

[41] T. D. De Gruijl, H. J. Bontkes, J. M. M. Walboomers et al., "Immune responses against human papillomavirus (HPV) type 16 virus-like particles in a cohort study of women with cervical intraepithelial neoplasia. I. Differential T-helper and IgG responses in relation to HPV infection and disease outcome," Journal of General Virology, vol. 80, no. 2, pp. 399-408, 1999.

[42] H. Hendel, S. Caillat-Zucman, H. Lebuanec et al., "New class I and II HLA alleles strongly associated with opposite patterns 
of progression to AIDS," Journal of Immunology, vol. 162, no. 11, pp. 6942-6946, 1999.

[43] M. R. Thursz, D. Kwiatkowski, C. E. M. Allsopp, B. M. Greenwood, H. C. Thomas, and A. V. S. Hill, "Association between an MHC class II allele and clearance of hepatitis B virus in the gambia," New England Journal of Medicine, vol. 332, no. 16, pp. 1065-1069, 1995.

[44] L. J. Fanning, J. Levis, E. Kenny-Walsh, M. Whelton, K. O'Sullivan, and F. Shanahan, "HLA class II genes determine the natural variance of hepatitis C viral load," Hepatology, vol. 33, no. 1, pp. 224-230, 2001.

[45] A. Hildesheim and S. S. Wang, "Host and viral genetics and risk of cervical cancer: a review," Virus Research, vol. 89, no. 2, pp. 229-240, 2002.

[46] B. D. Juran, E. J. Atkinson, J. J. Larson et al., "Carriage of a tumor necrosis factor polymorphism amplifies the cytotoxic T-lymphocyte antigen 4 attributed risk of primary biliary cirrhosis: evidence for a gene-gene interaction," Hepatology, vol. 52, no. 1, pp. 223-229, 2010.

[47] A. S. Pulickal, S. Hambleton, M. J. Callaghan et al., "Biliary cirrhosis in a child with inherited interleukin-12 deficiency," Journal of Tropical Pediatrics, vol. 54, no. 4, pp. 269-271, 2008.

[48] G. M. Hirschfield, M. E. Gershwin, R. Strauss et al., "Ustekinumab for patients with primary biliary cholangitis who have an inadequate response to ursodeoxycholic acid: a proof-ofconcept study," Hepatology, vol. 64, no. 1, pp. 189-199, 2016. 


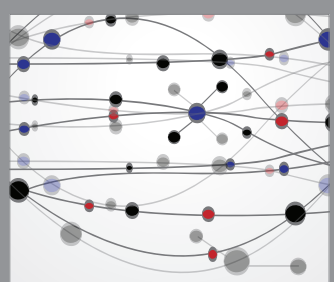

The Scientific World Journal
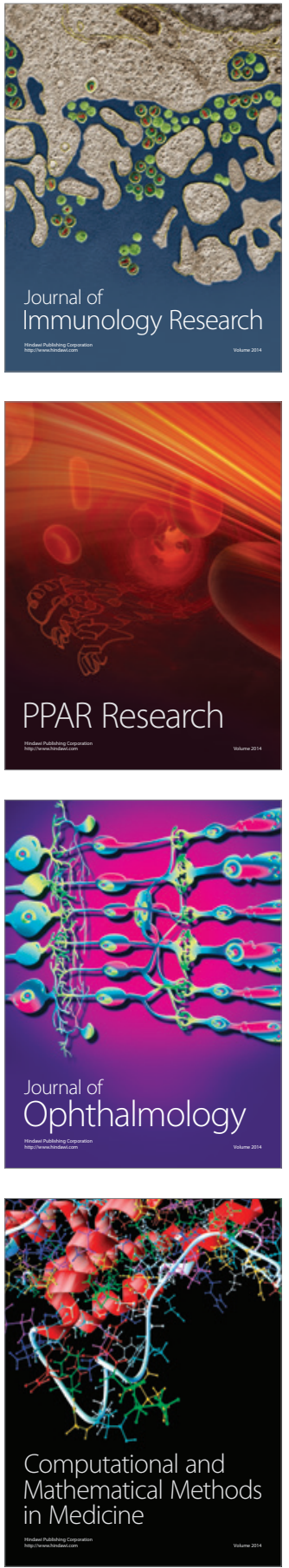

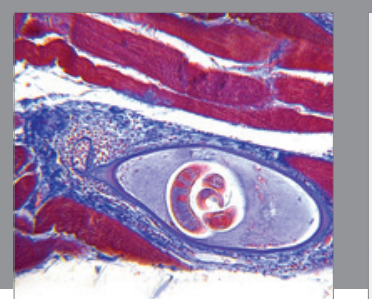

Gastroenterology Research and Practice
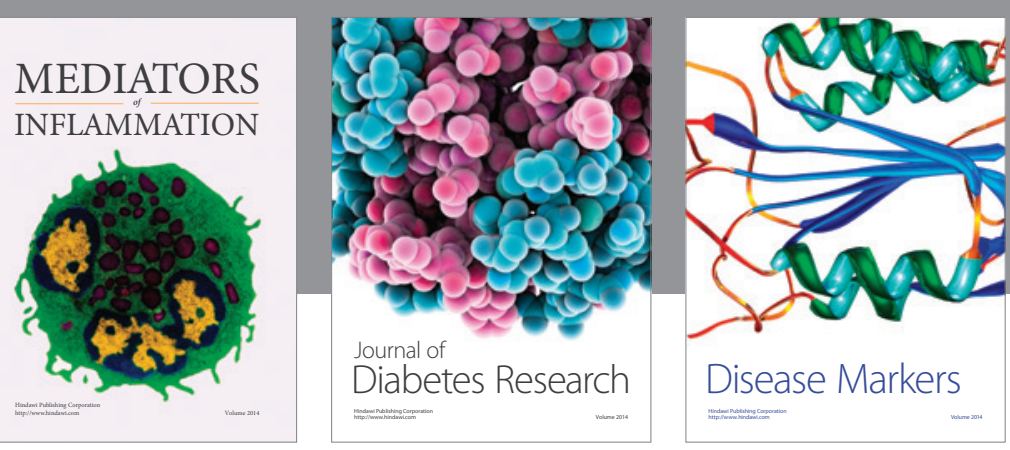

Disease Markers

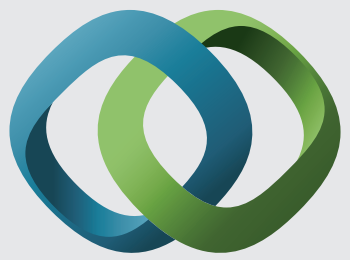

\section{Hindawi}

Submit your manuscripts at

https://www.hindawi.com
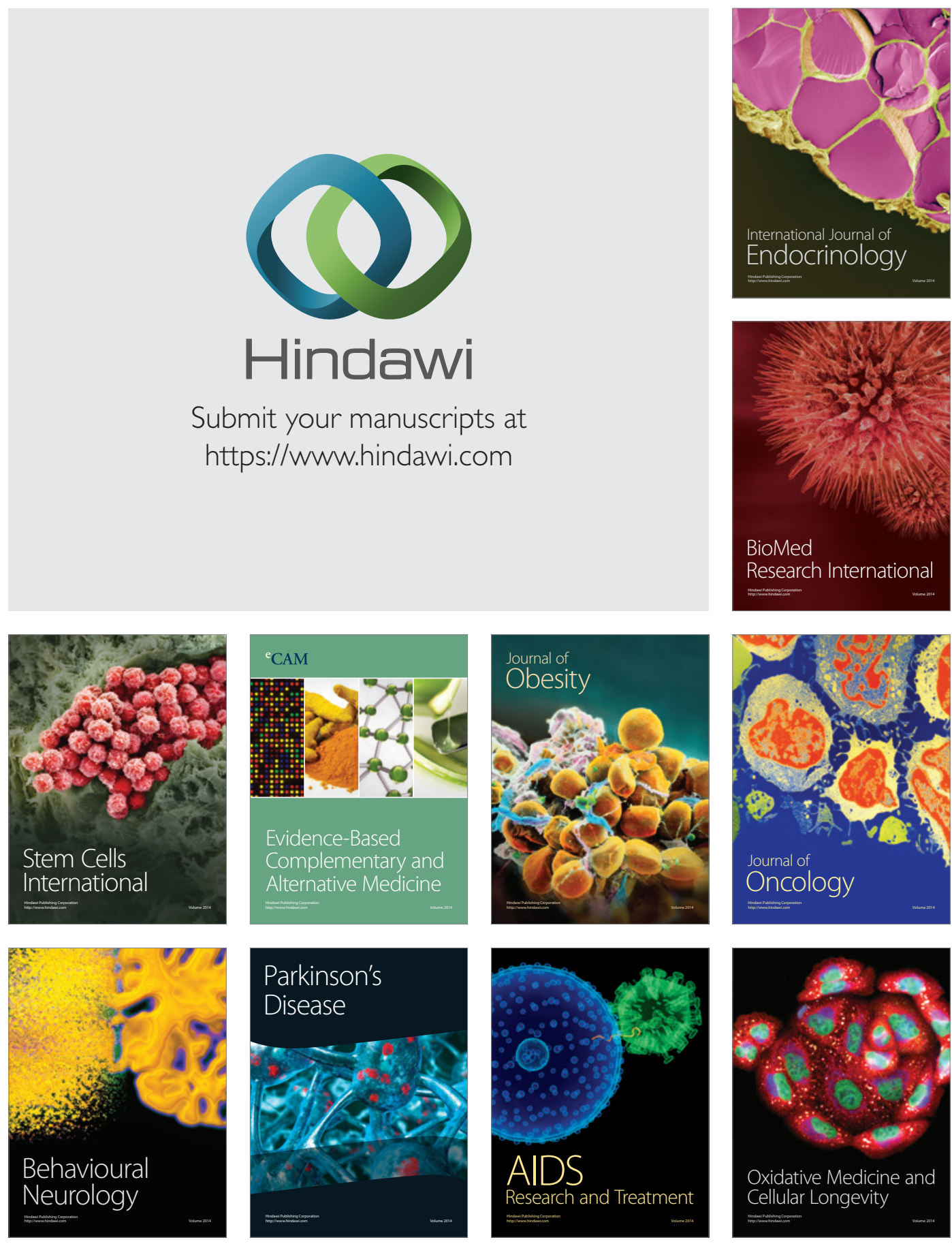